\title{
PCF INVESTIGATION TO IMPROVE THE PERFORMANCE OF TORA - BASED MANET AGAINST JAMMING ATTACKS
}

\author{
Sabbar Insaif Jasim ${ }^{1}$ \\ ${ }^{1}$ Technical Institute / Al-Dour
}

\begin{abstract}
Security in Mobile Ad Hoc Network became very important due to the nature of wireless communication between the nodes and the rapid movement of node which make Mobile Ad hoc Network vulnerable to Attackers. Jamming is a DoS attack's special category used in wireless networks. The attacker disrespects the medium access control (MAC) protocol and transmits on the shared channel; either periodically or continuously to target all or some communication, respectively. Distributed coordination function (DCF) and Point coordination function (PCF) are the two different media access control (MAC) mechanisms which are specified by the IEEE 802.11 standard. PCF can achieve higher throughput than DCF due to the nature of contention-free, therefore, this paper investigate the impact of PCF when integrated into the TORA - Based MANET and how it can improve the performance of the network. OPNET - Based simulation scenarios were created and the simulation was run and the results were collected which investigate that PCF provided a good functionality to improve deficiency caused by the Jammers this by increasing the throughput and decreasing the delay which is affected by the Jammers. PCF was a good improvement with different levels of Jammers' transmission power.
\end{abstract}

\section{KEYWORDS}

Network Protocols, Wireless Network, Mobile Network, Virus, Worms \&Trojon.

\section{INTRODUCTION}

In last few years, Mobile ad hoc network had gained a lot of attention due to its power constraints, security issues, network topology, dynamic independent, and limited range of each mobile host's wireless transmissions... etc [1]. Mobile Ad hoc Networks are peer to peer networks and represent a fully mobile infrastructure due to the wireless communication between mobile nodes in MANETs. MANET can be created and used at anytime, anywhere without any preexisting base station infrastructure and central administration [2]. Due to the nature of wireless communication between the nodes and the rapid movement of node, this make Mobile Ad hoc Network vulnerable to Attackers.

MANET's security is very low when compared to the wired network [3]. Due to the nature of contention-free, PCF (mechanism of Media Access Control of the IEEE 802.11 standard) can achieve higher throughput than the contention-based DCF and provide guarantee service [4]. This paper investigate how can PCF improve MANET - performance which is reduced by Jamming Attacks. OPNET Modeler (v14.5) as a simulation tool in this study to investigate PCF improvement. 
International Journal of Computer Science \& Engineering Survey (IJCSES) Vol.5, No.3, June 2014

\section{RELATED WORK}

Many Works had been done on the security issues together with the Jamming attacks of MANET. Ali Hamieh, Jalel Ben-Othman consider a particular class of DoS attacks called Jamming. and propose a new method of detection of Jamming attacks by the measurement of error distribution [5]. Tajinderjit Kaur and Sangeeta Sharma introduced that jamming attack in the networks having nodes with isotropic and directional antennas. The work's simulation results show that it is possible to minimize the effect of jamming attack by using different antenna patterns [6]. Jalel Ben-Othman, Ali Hamieh propose a new method to react at jamming attacks. The military has long dealt with jamming by using frequency-hopping spread spectrum communication [7]. Achint Gupta1, Dr. Priyanka V.J., and Saurabh Upadhyay have analyzed the effect of wormhole attack on AODV routing protocol based Mobile Ad-hoc Network using OPNET simulator using parameter like number of hops, delay, retransmission attempt, and data dropped [8].

\section{MOBILE Ad HOC NETWORK}

An ad-hoc network is formed when two or more stations come together form an independent network. Ad-hoc networks do not require any prior infrastructure, therefore, they are also termed as infrastructure-less networks [9] consisting of both fixed node and mobile nodes exchange data with each other without any centralised infrastructure or base station. The transitional node behaves like router to transmit data to nodes not in range [10]. Each node in the MANET having its own processing capability and energy resources and the mobile nodes are moving rapidly. MANET can be easily established in any emergency situations which can be used in disaster recovery, conferences, , emergency situation in hospitals, meetings, lectures [2].

Mobile Ad-hoc Network has a number of protocols which are classified as Reactive, Proactive and Hybrid for difference types of MANET such as (AODV, DSR, OLSR, TORA and GRP) [10].

\section{SECURITY ISSUES IN MANET}

In a MANET, nodes within ranges of each other's wireless transmission can communicate directly; however, nodes outside that range will depend on some other nodes to relay messages. An essential set of security mechanism must be encapsulated for any routing protocol to detect, prevent, and respond to security attacks. In order to investigate a reliable and secure ad-hoc network environment There are five major security goals. They are mainly: Authentication, Integrity, Confidentiality, Non-repudiation and Availability [11].

\subsection{MANET Attacks}

The threats for MANETs are classified as shown in Figure 1 [12] 


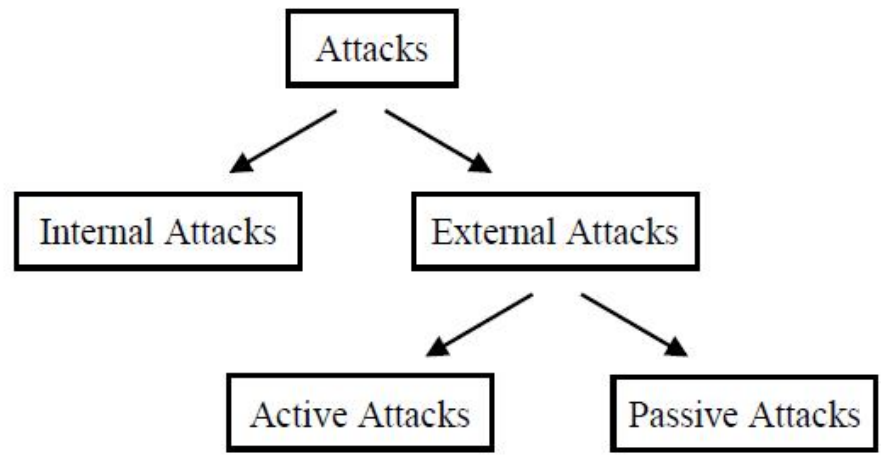

Figure 1 Types of MANET Attacks

Among attacks that are considered more severe, the attacks which create isolation of the nodes on the network which can result in denial-of-service and hence network collapses completely. Jamming Attack is the simplest form of such attacks which can block any current legitimate communication [13].

\subsection{Jamming Attacks}

Firstly one should know what jammer is. Jammer is defined as an individual who is intentionally obstructing the methods of legal wireless communication. It is treated as an active attacker depending upon its intentions and actions. Jamming is a DoS attack's special category used in wireless networks. Handling of Jamming attacks much harder than other attacks. The attacker disrespects the medium access control (MAC) protocol and transmits on the shared channel; either periodically or continuously to target all or some communication, respectively [13]. In fact, a wireless medium is shared in the mobile hosts in mobile ad hoc networks. A radio signal can be interfered or jammed, which causes the message to be corrupted or lost. The attacker with a powerful transmitter causes that the generated signal will be strong enough to crush the targeted signals and damage communications [5].

\section{POINT COORDination FunCtion}

Distributed coordination function (DCF) and Point coordination function (PCF) are the two different media access control (MAC) mechanisms which are specified by the IEEE 802.11standard. DCF is the basic MAC mechanism whereas PCF is built on top of DCF and provides contention-free media access. PCF can achieve higher throughput than the contentionbased DCF due to the nature of contention-free, and PCF provide guaranteed service which is important for real-time applications and PCF could also be used for non-real-time services which will be an attractive option for future wireless networks [4].

As described previously, PCF achieves higher throughput in network. This paper investigate the impact of PCF when integrated into the MANET and how it can improve the performance of the network.

\section{OPNET AS S SIMULATION TOOL}

A number of Simulation Based research model is available for students, researchers and commercial professionals in Computer Network Science. One of the popular simulations for networks is OPNET (Optimized Network Engineering Tools) Modeller which is widely used by 
different organisations and educational bodies. OPNET supports most of the effective MANET protocols. OPNET is a DES based simulator which supports parallel processing [10]. OPNET Modeler environment includes tools for all phases of a study, including data collection, data analysis, model design, simulation to support all network types. OPNET Modeler directly parallel the structure of real networks, equipment, and protocols based on a series of hierarchical editors [11].

\subsection{OPNET-Based Simulation Setup}

The simulation setup consists of number of OPNET's scenarios, each scenario consists of number of objects from OPNET's object Palette which are configured to form the proposed network with ad-hoc configuration and TORA Routing protocol as follows:

\subsubsection{Scenario 1: MANET without Jammers}

This scenario consists of number of wireless stations (mobile nodes) named (wlan_wkstn_adv) in ad hoc connection as follows: this scenario shown in Figure 2.

No. of work stations $=20$

The transmission power of each station $=0.005 \mathrm{~W}$

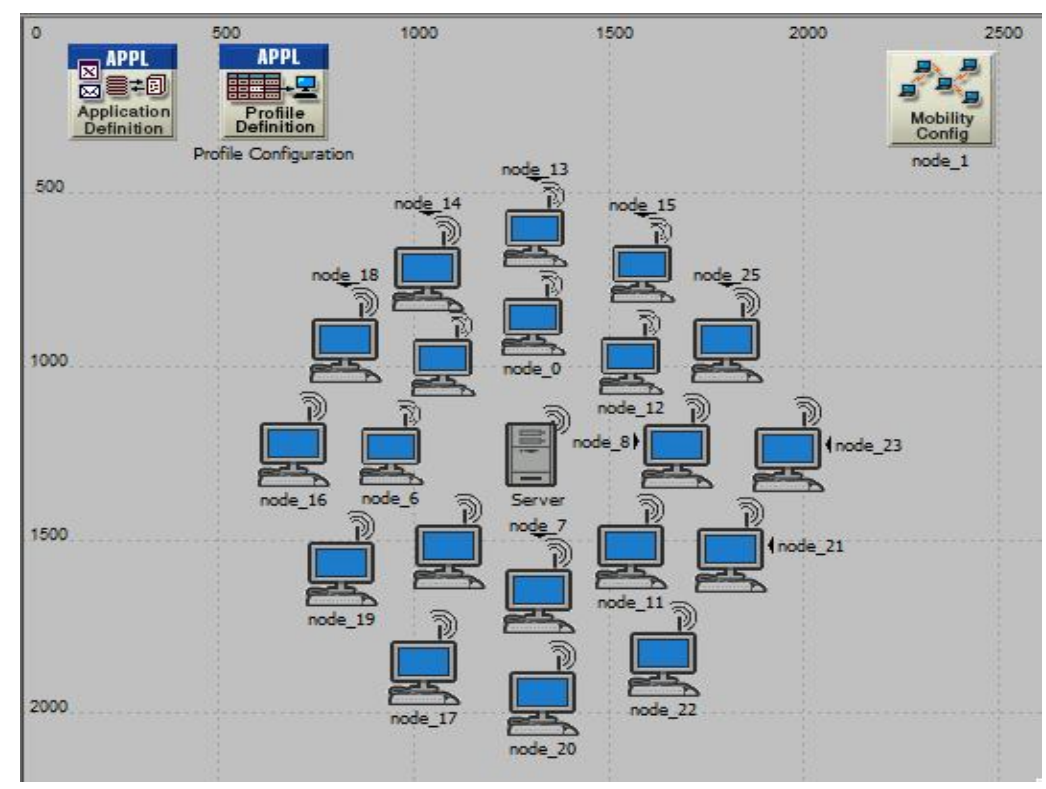

Figure 2 MANET without Jammers

\subsubsection{Scenario 2: MANET with Jammers}

Four pulsed Jammers named (jam_pulsed) added to scenario 1 (MANET without Jammers) as shown in Figure 3.

No. of work stations $=20$

The transmission power of each station $=0.005 \mathrm{~W}$

No. of Jammers $=4$

The transmission power of each Jammer $=0.001 \mathrm{~W}$ 
International Journal of Computer Science \& Engineering Survey (IJCSES) Vol.5, No.3, June 2014

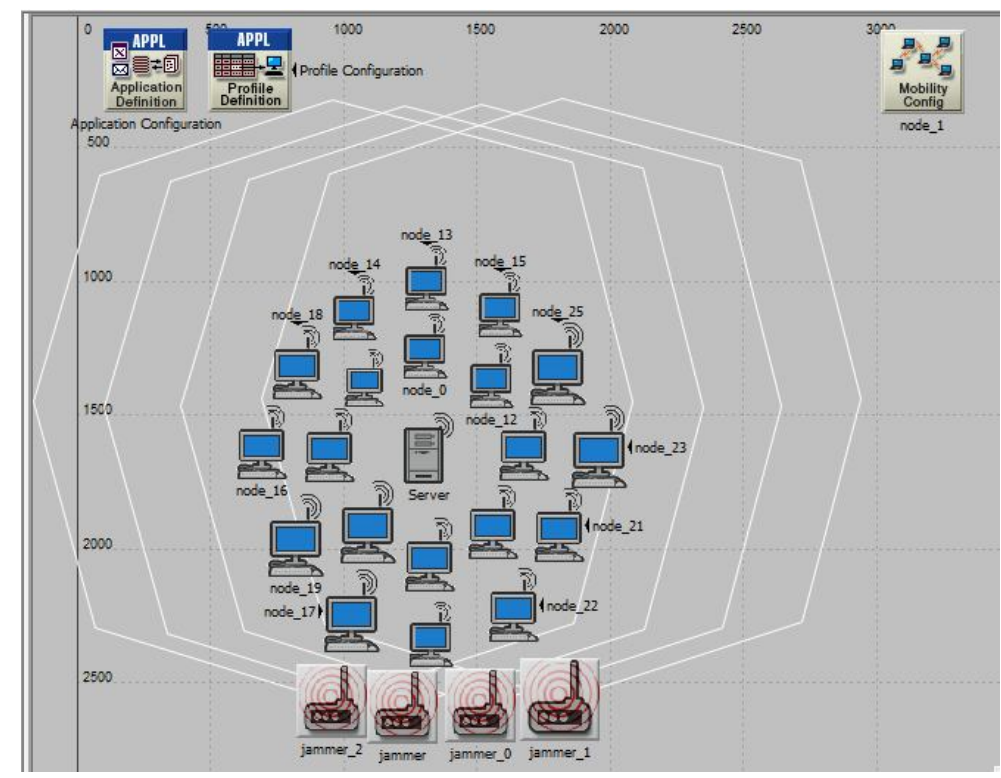

Figure 3 MANET with Jammers

\subsubsection{Scenario 3: MANET with Jammers and enabled PCF}

In this scenario, PCF was enabled in four selected guard nodes among the red lines shown in Figure 4 to show how the PCF could improve the performance of (MANET with Jammers)to reduce the impact of Jammers on the performance of network in terms of (delay, throughput, .....). No. of work stations $=20$

The transmission power of each station $=0.005 \mathrm{~W}$

No. of Jammers $=4$

The transmission power of each Jammer $=0.001 \mathrm{~W}$

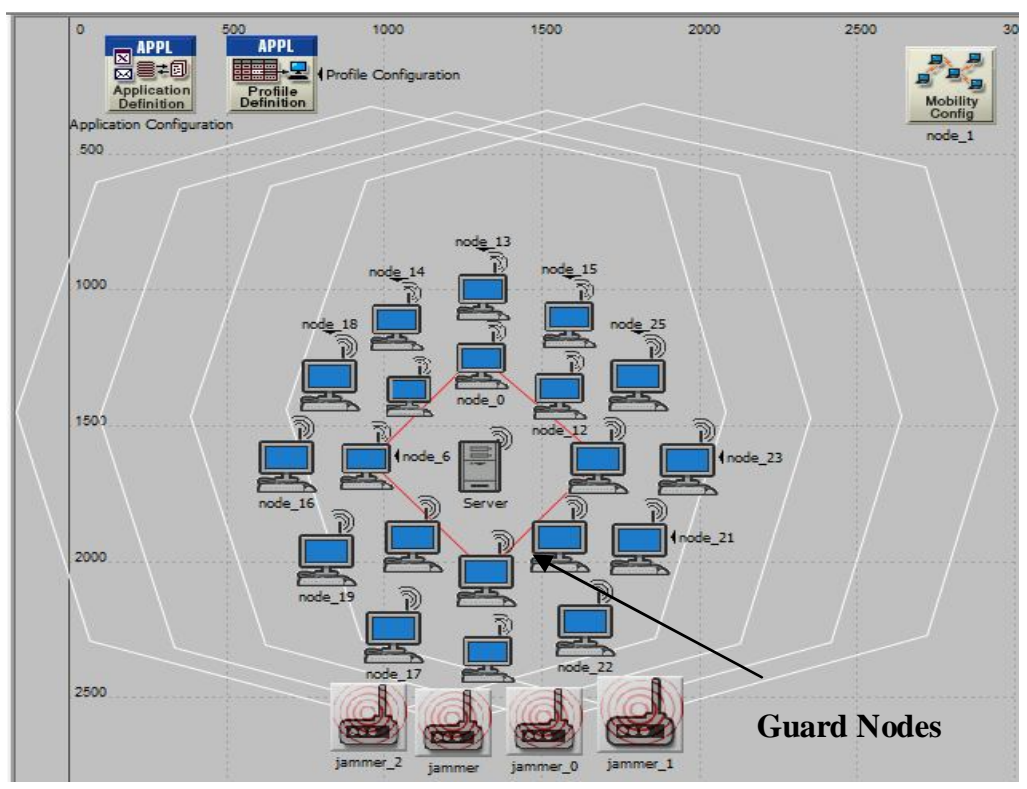

Figure 4 MANET with Jammers and enabled PCF 


\subsubsection{Scenario 4: MANET with Jammers}

This scenario consists of 20 workstations in ad hoc connection and four jammers were applied to the network as shown in Figure 5. In this scenario, transmission power of jammer was increased.

No. of work stations $=20$

The transmission power of each station $=0.005 \mathrm{~W}$

No. of Jammers $=4$

The transmission power of each Jammer $=0.01 \mathrm{~W}$

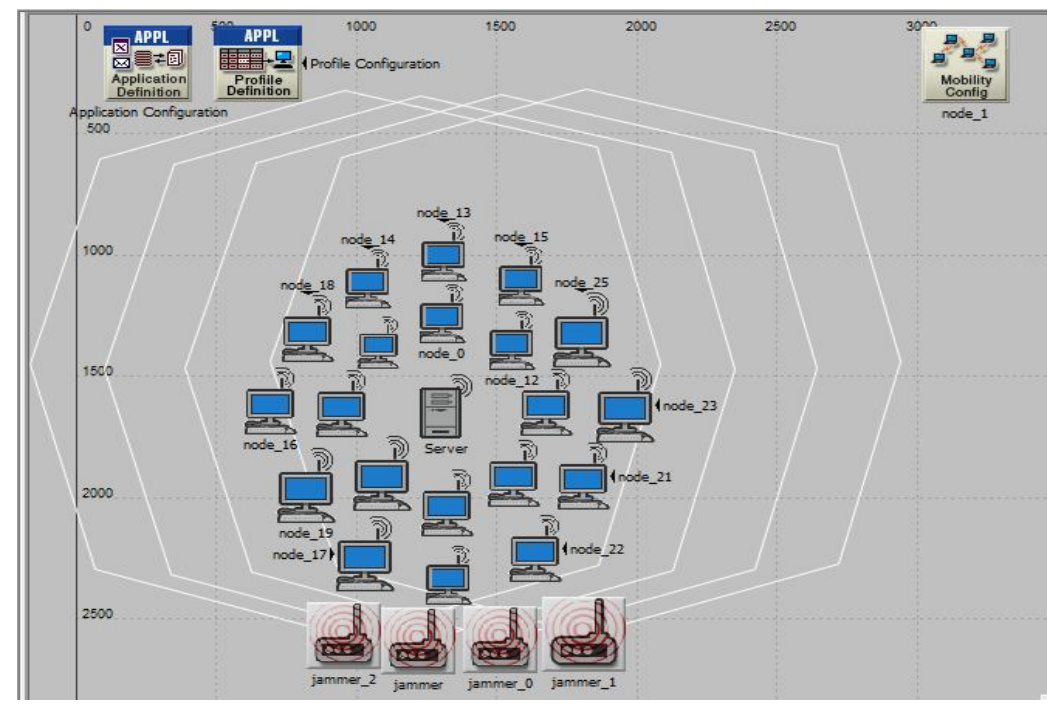

Figure 5 MANET with Jammers

\subsubsection{Scenario 5: MANET with Jammers and enabled PCF}

In this scenario, PCF was enabled in four selected guard nodes among the red lines shown in Figure 6 to show how the PCF could improve the performance of (MANET with Jammers)even if the transmission power of the Jammer was increased to $(0.01 \mathrm{~W})$ in order to reduce the impact of Jammers on the performance of network in terms of (delay, throughput, .....).

No. of work stations $=20$

The transmission power of each station $=0.005 \mathrm{~W}$

No. of Jammers $=4$

The transmission power of each Jammer $=0.01 \mathrm{~W}$ 
International Journal of Computer Science \& Engineering Survey (IJCSES) Vol.5, No.3, June 2014

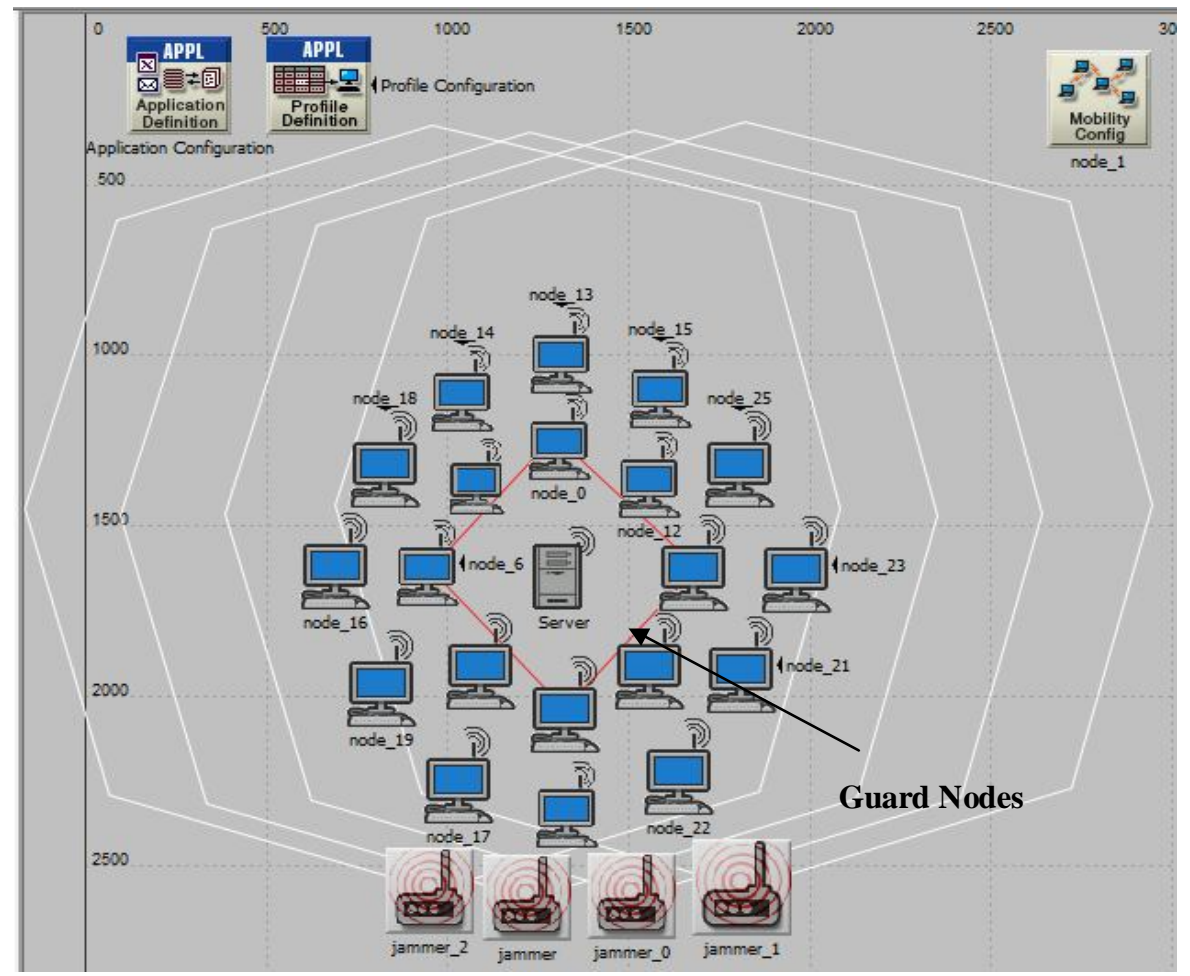

Figure 6 MANET with Jammers and enabled PCF

\section{RESULTS AND DISCUSSION}

Discrete Event Statistics were chosen for each scenario, these statistics include (throughput, delay, data dropped. The simulation was run for 15 minutes and the results were collected as follows:

\section{Transmission Power of Jammer=0.001 W}

1- Throughput: Represents the total number of bits (in bits/sec) forwarded from wireless LAN layers to higher layers in all WLAN nodes of the network.

Throughput can be represented mathematically as in eq.(1) (Naveen Bilandi, et al., 2010)

Throughput $=\frac{\text { Numberof delivered packet } * \text { Packet size } * 8}{\text { Totalduration of simulation }}$

Throughput for three scenarios (MANET without Jammers, MANET with Jammers, MANET with Jammers and enabled PCF) were shown in Figure 7. 
International Journal of Computer Science \& Engineering Survey (IJCSES) Vol.5, No.3, June 2014

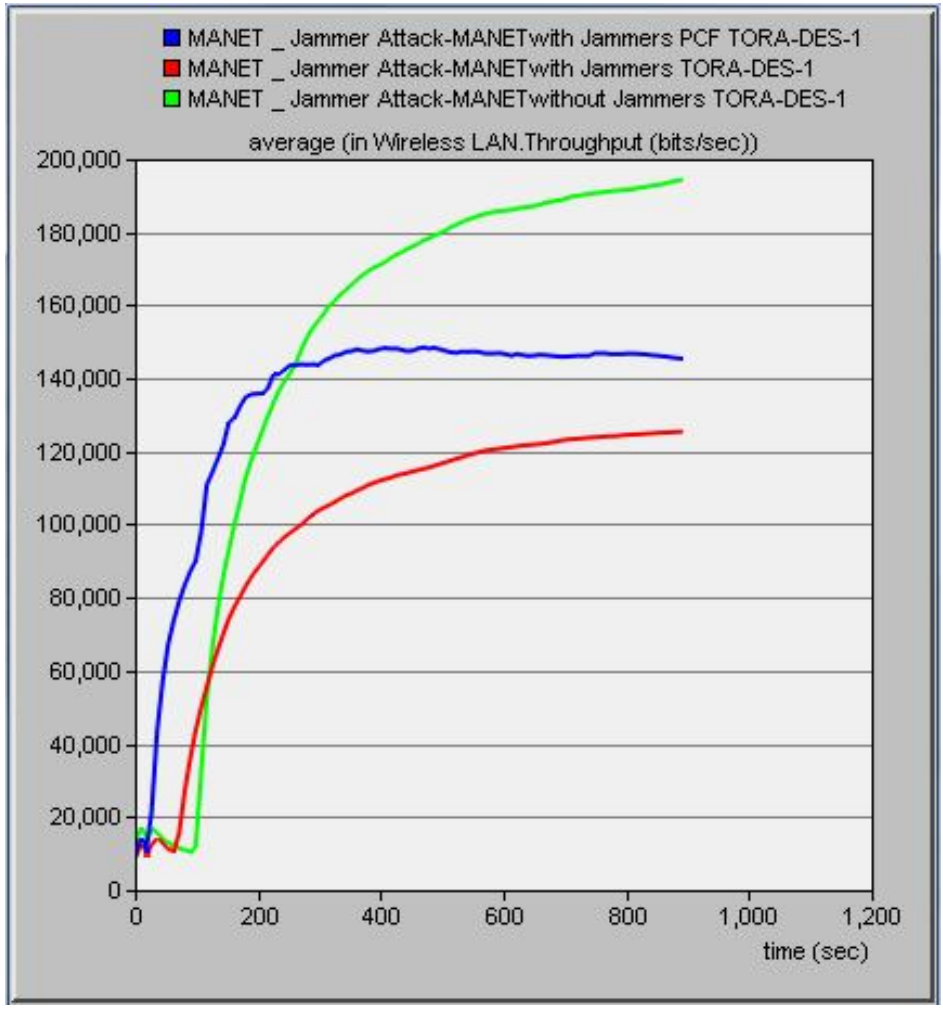

Figure 7 Throughput

As shown previously, the existence of Jammers would reduce throughput from 194,399.4 bits/sec to $125,428.5$ bits/sec. PCF which was enabled in the selected guard nodes can improve throughput to $145,367.1 \mathrm{bits} / \mathrm{sec}$.

2- Delay: Represents the end to end delay of all the packets received by the wireless LAN MACs of all WLAN nodes in the network and forwarded to the higher layer.

Delay can be represented mathematically as in eq.(2) (Naveen Bilandi et al., 2010)

$\mathrm{d}_{\text {end-end }}=\mathrm{N}\left[\mathrm{d}_{\text {trans }}+\mathrm{d}_{\text {prop }}+\mathrm{d}_{\text {proc }}\right]$

Where:

$\mathrm{d}_{\text {end-end }}=$ End to end delay

$\mathrm{d}_{\text {trans }}=$ Transmission delay

$\mathrm{d}_{\text {prop }}=$ Propagating delay

$\mathrm{d}_{\text {proc }}=$ Processing delay

Delay for three scenarios (MANET without Jammers, MANET with Jammers, MANET with Jammers and enabled PCF) were shown in Figure 8. 
International Journal of Computer Science \& Engineering Survey (IJCSES) Vol.5, No.3, June 2014

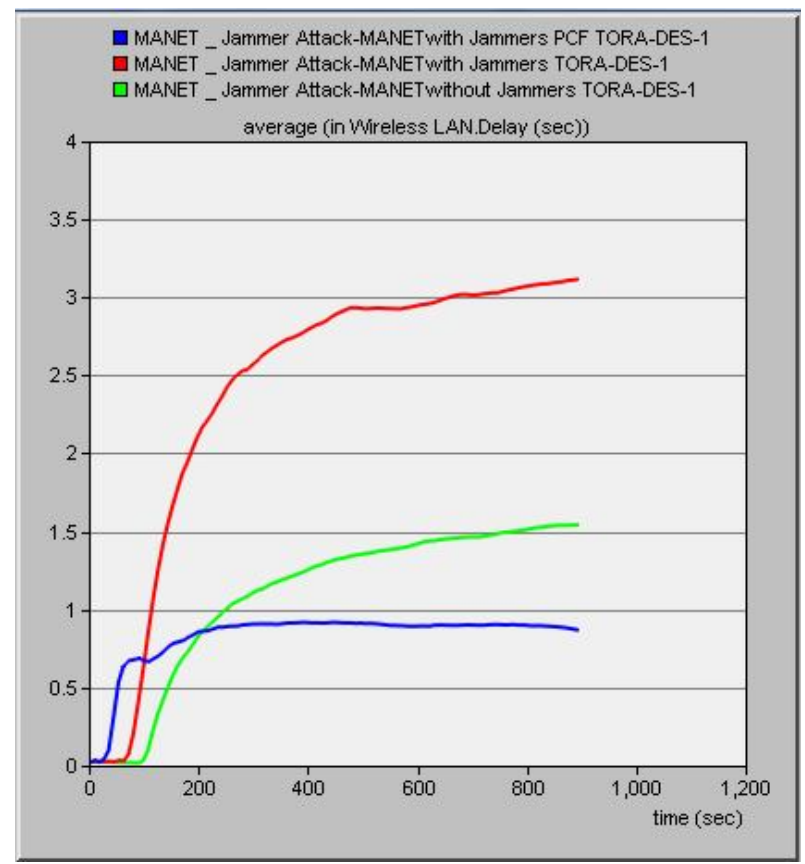

Figure 8 Delay

With Jammers, the delay was increased from $1.543 \mathrm{sec}$ to $3.115 \mathrm{sec}$. PCF which was enabled in the selected guard nodes can improve the network performance by decreasing delay to $0.87 \mathrm{sec}$ which was very good improvement in terms of delay.

3- Traffic Received (Bytes/sec): Average bytes per second forwarded to the HTTP applications by the transport layers in the network for for three scenarios (MANET without Jammers, MANET with Jammers, MANET with Jammers and enabled PCF) were shown in Figure 9

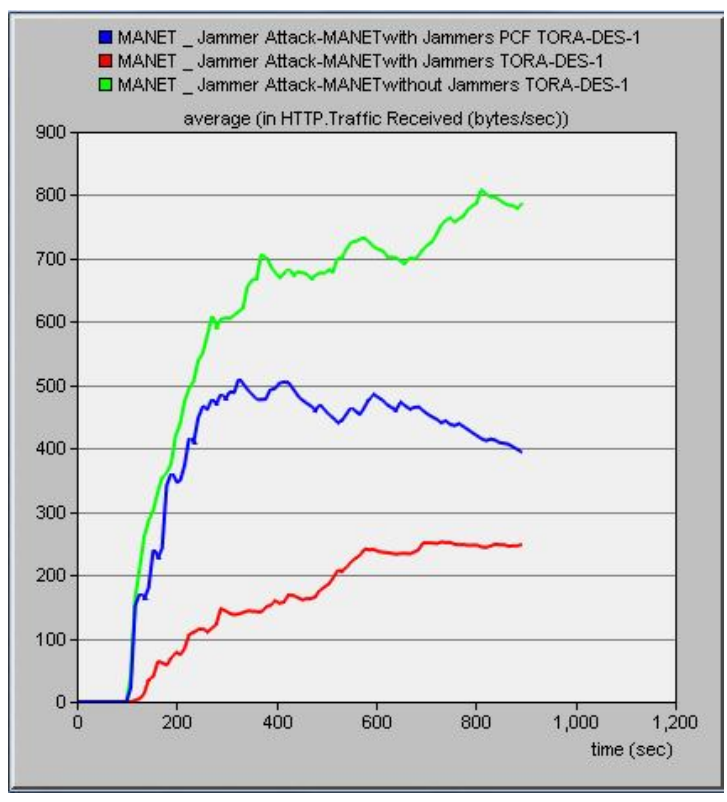

Figure 9 Delay 
International Journal of Computer Science \& Engineering Survey (IJCSES) Vol.5, No.3, June 2014

Jammers reduced traffic received from 786.74 bytes/sec to 248.12 bytes/sec. PCF enabled in the guard nodes would increased Traffic received to $394.23 \mathrm{bytes} / \mathrm{sec}$.

\section{Transmission Power of Jammer=0.01 W}

Throughput, delay and Traffic received for three scenarios (MANET without Jammers, MANET with Jammers, MANET with Jammers and enabled PCF) Transmission Power of Jammer $=0.01$ W were shown in Figure 10, Figure 11 and Figure 12 respectively.

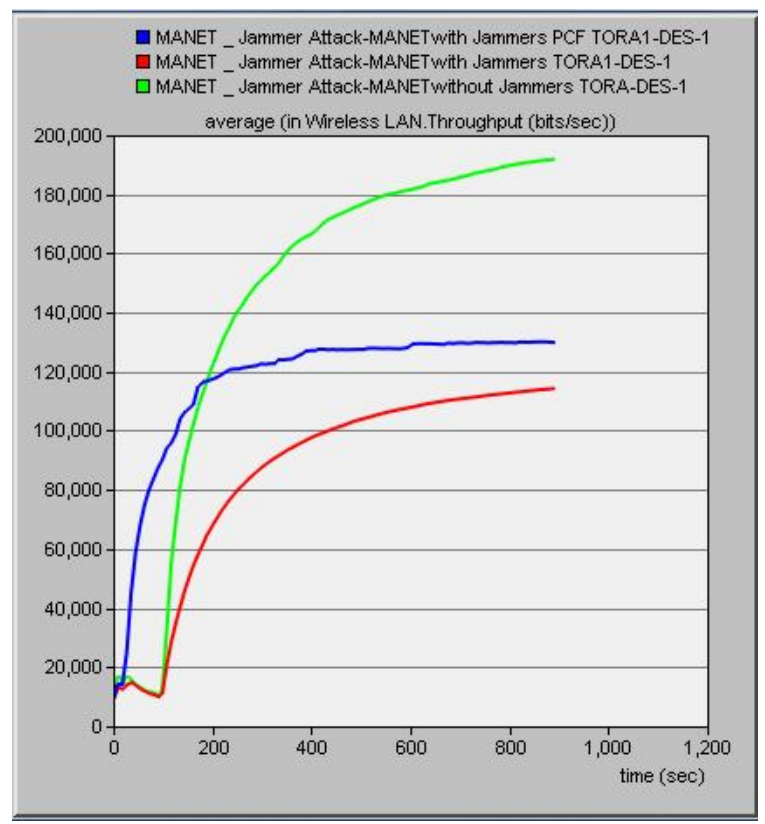

Figure 10 Throughput

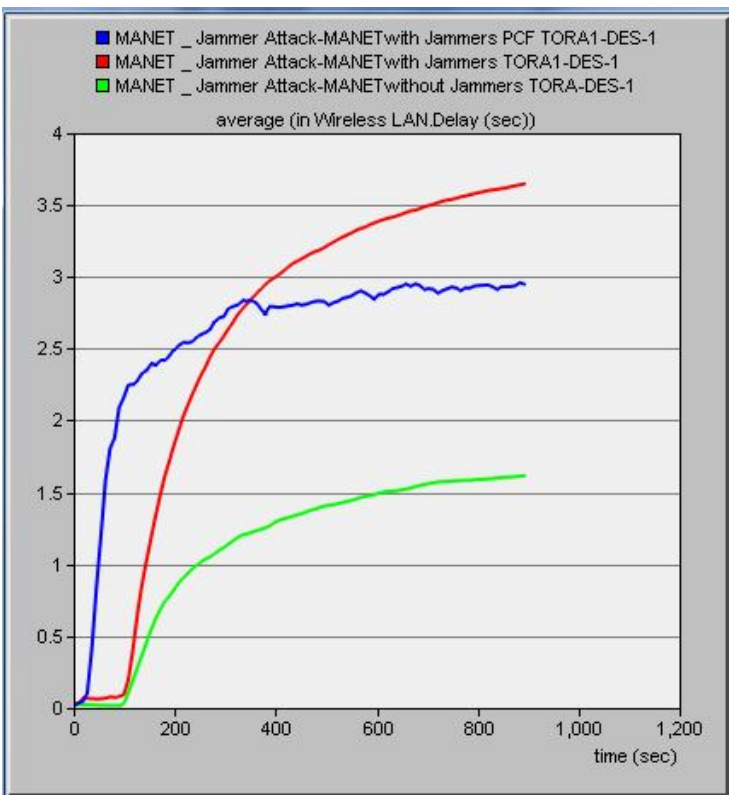

Figure 11 Delay 


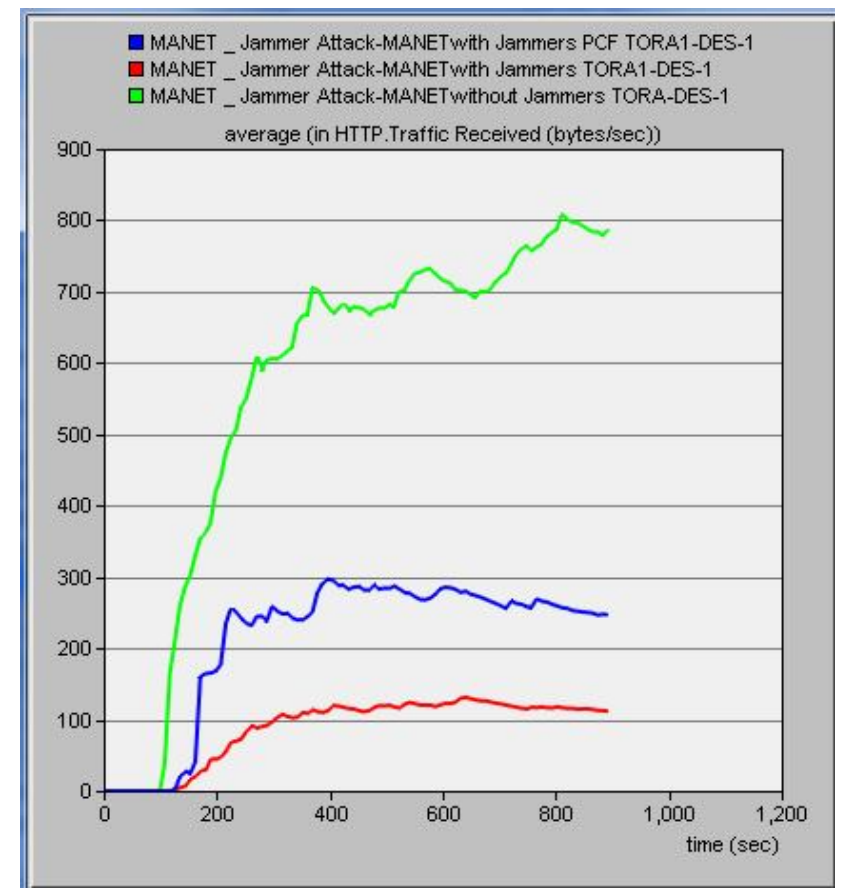

Figure 12 Traffic received

PCF achieved good improvement even when transmission power of jammers was increased to $0.01 \mathrm{~W}$. this was achieved by increasing throughput and decreasing delay as shown in above figures (Figure 10, Figure 11 and Figure 12) respectively.

\section{Conclusions}

Mobile ad hoc network has been a challenging research area for the last few years, Due to the nature of wireless communication between the nodes and the rapid movement of node, and sharing of wireless medium, this make Mobile Ad hoc Network vulnerable to Attackers so that a radio signal can be jammed or interfered, which causes the message to be corrupted or lost. Jammer will reduce the performance of the network by decreasing the throughput and increasing delay. In the Jammer based proposed network, PCF which achieved higher throughput was enabled into the network in four guard nodes in order to improve the performance of the proposed network. The OPNET Modeller (v14.5) was used as a simulation tool for this study. After the statistics were chosen and the simulation was run for 15 minutes, the results were collected and showed that PCF gave a good improvement to increase throughput and traffic received which were reduced by the Jammers and decrease the delay which was increased by the Jammers. PCF achieved somewhat a good improvement even when the transmission power of the Jammers was increased to $0.01 \mathrm{~W}$. PCF provided a good functionality to improve deficiency caused by the Jammers. This performance study was studied in terms of some parameters for TORA routing protocol. Other MANET routing protocols such as (AODV, DSR, DSDV, OLSR, GRP) could be taken with other parameters for further studies such as (no of hops per route, packet dropped, route error sent, Acknowledgment and Acknowledgement request sent, retransmission attempts and buffer overflow). 
International Journal of Computer Science \& Engineering Survey (IJCSES) Vol.5, No.3, June 2014

\section{REFERENCES}

[1] Abhay Kumar Rai, Rajiv Ranjan Tewari, Saurabh Kant Upadhyay, "Different Types of Attacks on Integrated MANET-Internet Communication", International Journal of Computer Science and Security (IJCSS) Volume (4): Issue (3), 2010, pp. 265-274.

[2] Jahangir khan, Dr.syed Irfan Hyder, Dr.Syed Malek and Fakar Duani syed Mustafa, "Modeling and Simulation Of Dynamic Intermediate Nodes And Performance Analysis in MANETS Reactive Routing protocols", International Journal of Grid and Distributed Computing, Vol. 4, No. 1, March 2011, pp. 31-56.

[3] 1Kishore Dasari, 2Tammineedi Venkata Satya Vivek, "Security Issues in Mobile Ad-Hoc Networks", IJCST Vol. 4, Iss ue Spl - 4, Oct - Dec 2013, pp. 104-107.

[4] Hao zhu and Guohong CAO, "On Improving the Performance of IEEE 802.11 with Relay-Enabled PCF", Mobile Networks and Applications 9, Kluwer Academic Publishers. Manufactured in The Netherlands., 2004, pp. 423-434.

[5] Ali Hamieh, Jalel Ben-Othman, "Detection of Jamming Attacks in Wireless Ad Hoc Networks using Error Distribution”, 978-1-4244-3435-0/09 IEEE, 2009.

[6] Tajinderjit Kaur, Sangeeta Sharma, "Mitigating the Impact of Jamming Attack by Using Antenna Patterns in MANET", VSRD International Journal of CS \& IT Vol. 2 (6), 2012, pp. 437-445.

[7] Jalel Ben-Othman, Ali Hamieh, "Defending Method Against Jamming Attack in Wireless Ad Hoc Networks", The 5th IEEE International Workshop on Performance and Management of Wireless and Mobile Networks (P2MNET 2009), Zürich, Switzerland; 20-23 October 2009, pp.758-762.

[8] Achint Gupta , Dr. Priyanka V J , Saurabh Upadhyay , "Analysis of Wormhole Attack in AODV based MANET Using OPNET Simulator”, International Journal of Computing, Communications and Networking, 1(2), September - October 2012, pp. 63 - 67.

[9] Ajay Dureja, Aman Dureja and Meha Khera, "IEEE 802.11 Based MAC Improvements for MANET", IJCA Special Issue on “Mobile Ad-hoc Networks”, MANETs, 2010, pp. 54 - 57.

[10] Md Maruf Ilahi, "Analyzing MANET Routing Performance Using OPNET Simulation”, A dissertation submitted toCity of London Collegein partial fulfilment of the requirements of Degree ofMasters in Computing (Computer Networks)Awarded by University of WalesSupervisor: Dr Sahar Al-SudaniAugust, 2011.

[11] Pradip M. Jawandhiya, Mangesh M. Ghonge, Dr. M.S..Ai and Prof. J.S. Deshpande "A Survey of Mobile Ad Hoc Network Attacks", International Journal of Engineering Science and Technology, Vol. 2(9), 2010, 4063-4071.

[12] Lathies Bhasker T, “ A SCOPE FOR MANET ROUTING AND SECURITY THREATS”, ICTACT JOURNAL ON COMMUNICATION TECHNOLOGY, VOLUME: 04, ISSUE: 04, DECEMBER 2013, pp. $840-848$.

[13] Faraz Ahsan, Ali Zahir, Sajjad Mohsi, Khalid Hussain, "Survey on survival approaches in wireless network against jamming attack", Journal of Theoretical and Applied Information Technology, 15th. Vol. 30 No.1, August 2011, pp. 55 - 67.

[14] Available online, http:://www.opnet.com.

\section{Authors}

Sabbar Insaif Jasim had M.Sc. degree in Computer Control Engineering from Baghdad university. He received B.Sc. degree from university of Baghdad, collage of engineering in Electrical Engineering . He is a lecturer in Department of Electronics, Al-Dour Technical Institute, He presents many papers in national and internationl journals and participate in number of conferences.

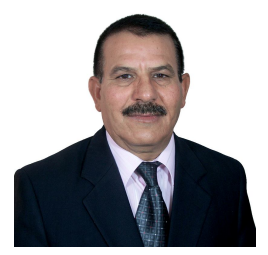

